\title{
Damage of White Matter in the Parietal Lobe Results in Anomic Alexia of Kana
}

\author{
Nobusada Shinoura, Yuichi Suzuki, Masanobu Tsukada, Ryozi Yamada, Yusuke Tabei, \\ Tomoyuki Koizumi, Kazuo Yagi
}

Can. J. Neurol. Sci. 2010; 37: 113-117

The reading and writing of two different types of Japanese words, "kana" and "kanji", are processed by different neural pathways. For example, kana are phonographic representations of words that are processed though the dorsal route related to phonological processing in a manner similar to that for words of phonetic languages (e.g., English). Investigators have reported that the left angular gyrus is critical for the process of writing kana $^{1,2}$. By contrast, kanji are logographic representations of words and are processed through the ventral route related to visual processing in a manner similar to that for logographic languages (e.g., Chinese) ${ }^{3-5}$. Investigators have reported that the left posterior inferior or middle temporal cortex is critical for the process of reading or writing kanji ${ }^{2,6-8}$.
Patients with alexia and anomic aphasia, namely anomic alexia for kanji, present with lesions that correspond to the middle temporal gyrus and its subcortical area ${ }^{9}$. Conversely, alexia and anomic aphasia for English are induced by lesions of the left occipital and medial temporal lobe ${ }^{10}$. To date, there are no reports that characterize lesions responsible for anomic alexia for kana and the relationship between reading of kana and neural tracts remain unclear.

Newer, noninvasive diagnostic modalities have enabled characterization of the neuroanatomic distribution of various language functions within the human brain. Of these new diagnostic modalities, diffuse tensor imaging (DTI) provides information regarding the trajectories of cerebral white matter
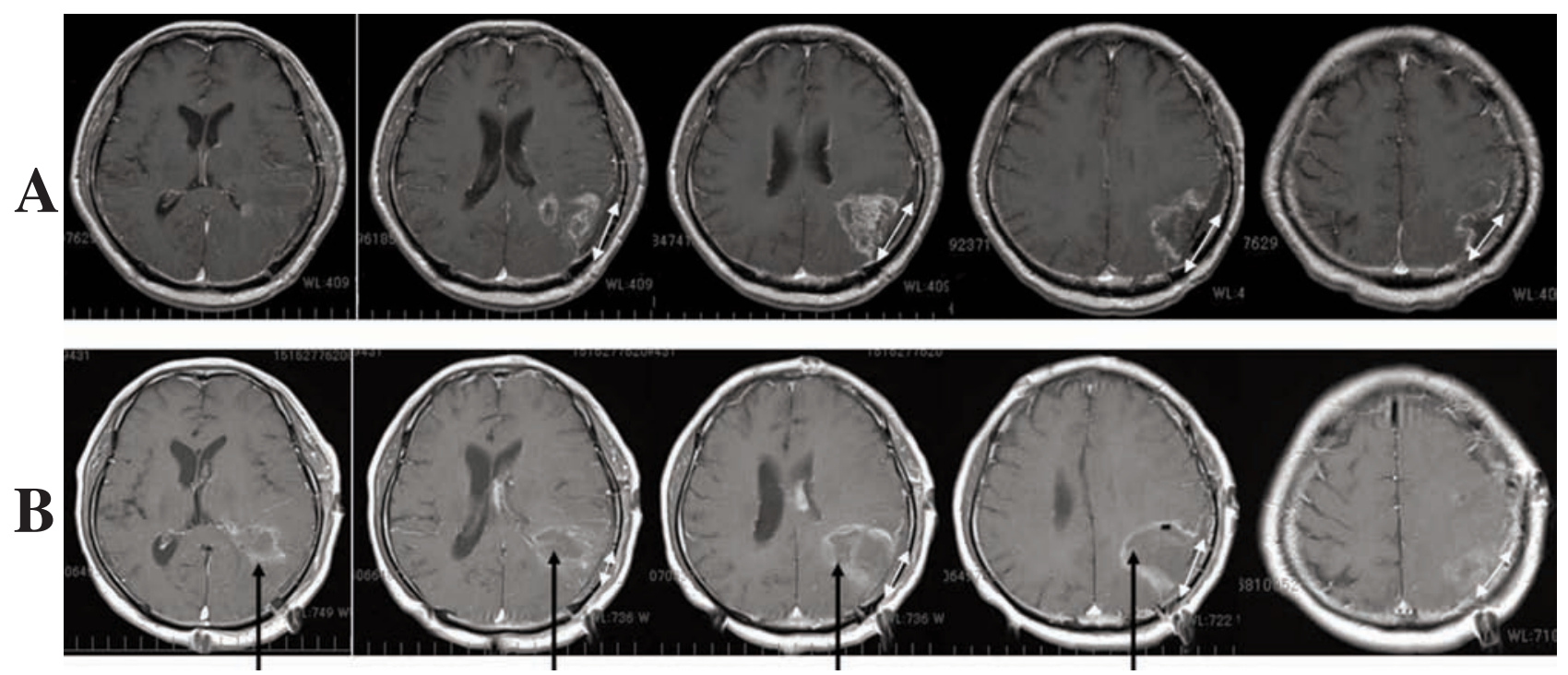

Figure 1: MRI (Panel A: before second surgery, Panel B: after second surgery) shows a heterogeneous and enhancing lesion in the left inferior parietal lobe. When comparing Fig. $1 \mathrm{~A}$ and Fig. $1 \mathrm{~B}$ quantitatively, the brain tissue removed by surgery was mainly limited to deep white matter beside the occipital horn of lateral ventricle, and not the cortex. This is supported by the fact that the postoperative width of the tumor cavity in the brain surface (i.e., the cortex) was the same or less than that before surgery (white arrow with double head), while the postoperative depth and width of the tumor cavity in the deep white matter after surgery was much larger than that before surgery (black arrow).

From the Departments of Neurosurgery (NS, RY, YT, TK) and Rehabilitation (MT), Komagome Metropolitan Hospital; Department of Radiologic Technology (YS, KY), Tokyo Metropolitan University of Health Sciences, Tokyo, Japan.

Received June 12, 2009. Final Revisions Submitted August 17, 2009.

Correspondence to: Nobusada Shinoura, Department of Neurosurgery, Komagome Metropolitan Hospital, 3-18-22 Hon-Komagome, Bunkyo-ku, Tokyo, 113-8677, Japan. 
tracts such as the inferior longitudinal fasciculus (ILF), inferior fronto-occipital fasciculus (IFOF), superior longitudinal fasciculus (SLF), including the arcuate fasciculus, or the occipital-callosal fibers (OCF) that run within the left temporal, parietal or occipital lobes ${ }^{11}$. Previous studies have reported that impairment of the left arcuate fasciculus results in impairment of reading ${ }^{12}$. The present study describes a case of DTI-documented disruption of deep white matter in the inferior parietal lobe (IPL) including the dorsal OCF that was associated with severe anomic alexia of kana.

\section{METHODS}

\section{Patient}

A 67-year-old male with a glioblastoma (diameter $=\sim 5 \mathrm{~cm}$ ) in the left parietal lobe presented for evaluation with right hemiparesis and sensory aphasia. In August 2008, the patient underwent surgical resection of the tumor. However, postoperative magnetic resonance imaging (MRI) demonstrated residual brain tumor and enhancement within the left parietal region (Figure 1A). In November 2008, the patient experienced abrupt onset of decreased level of consciousness, worsening right hemiparesis and sensory aphasia due to bleeding from the brain tumor. The patient underwent repeat surgery for tumor resection, and postoperative MRI showed completed tumor removal (Figure 1B). Postoperatively, the patient's level of consciousness returned to baseline. Quantitative analysis of Figure 1 suggests that the resected brain tissue was mainly limited to the deep white matter, not the cortex, since the width of the tumor cavity in the cortex after surgery remained the same or was reduced in comparison with that before surgery (white arrow) while the postoperative depth and width of the tumor cavity in the deep white matter was markedly larger than that before surgery (black arrow).

\section{DTI analysis}

Diffuse tensor imaging and image analysis were performed as previously described ${ }^{13}$. Briefly, standard imaging gradients were used with a maximum strength of $22 \mathrm{mT} / \mathrm{m}$ and a slew rate of 77 $\mathrm{mT} / \mathrm{m} / \mathrm{ms}$. All data were acquired using a birdcage head coil. The DTI acquisition sequence was a single-shot spin-echo echo planar imaging (EPI), with the following parameters: TE, 98.6 ms; acquisition matrix, $128 \times 128$; and field of view, $24 \mathrm{~cm} \times 24$ $\mathrm{cm}^{14}$. Contiguous 4-mm-thickness slices were obtained, covering the whole brain, with a b value of $1000 \mathrm{~mm}^{2} / \mathrm{s}$ in 42 noncollinear directions. Reconstructed voxel size was $1.88 \times 1.88 \times 4.00 \mathrm{~mm}^{3}$. DTI acquisition time for a total of 61 images was approximately ten min. Diffusion tensor eigenvalues $\left(\lambda_{1}, \lambda_{2}, \lambda_{3}\right)$ and eigenvectors $\left(\varepsilon_{1}, \varepsilon_{2}, \varepsilon_{3}\right)$ were calculated from DTI data, and fractional anisotropy (FA) maps were generated according to the

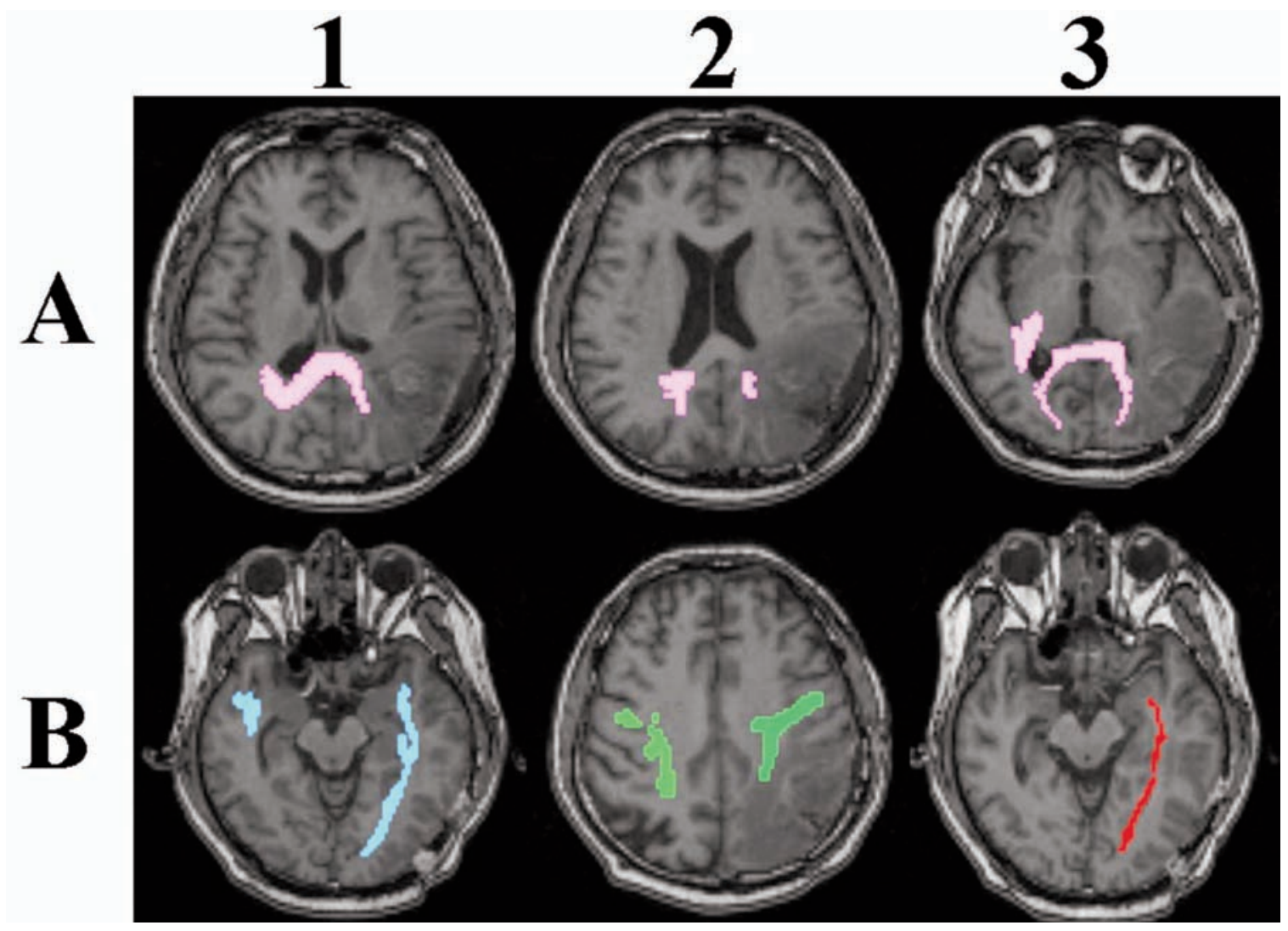

Figure 2: DTI images: Axial views before second surgery. The dorsal OCF (Panel A-1, A-2; pink), ventral OCF (Panel A-3; pink), ILF (Panel B-1; light blue), SLF (Panel B-2; green) and IFOF (Panel B-3; red) were constructed as described in the Methods section. All neural tracts on the left side were intact before surgery. 
Tensorlines algorithm ${ }^{15}$, as a combination of the Tensor Deflection algorithm at low FA and Streamlines tracking at high FA $^{16}$, using DTI Analyzer (IDL ver5.6; Research Systems). Stopping criteria (threshold 0.2) was used for analysis. The patient provided informed consent to undergo DTI. The ILF was constructed using the ROI of the white matter of the coronal section around the inferior horn of the lateral ventricle at the seed point and parieto-occipital sulcus at the target point in which the tracts ran from posterior to anterior; the uncinate fibers and the fibers that ran to the opposite side of the corpus callosum were deleted $^{11}$. The IFOF was constructed using the ROI of the external capsule of the coronal section near the anterior horn of the lateral ventricle in which the tracts ran from posterior to anterior; again, the uncinate fibers and the fibers that ran to the opposite side of corpus callosum were deleted. The SLF was constructed using the ROI of the white matter of the coronal section, which showed a triangular shape just lateral to the corticospinal tract near the anterior horn of the lateral ventricle in which the tracts ran from posterior to anterior. The OCF was constructed using the ROI of the splenium.

\section{Neuropsychological testing}

The Japanese Standard Language Test of Aphasia was performed to measure language ability before and after the second surgery ${ }^{17}$. Object naming, auditory kana or kanji
Table: Summary of pre- and postoperative test results for language function

\begin{tabular}{c|c|c}
\hline Standardized test & $\begin{array}{c}\text { Before } \\
\text { surgery (\%) }\end{array}$ & $\begin{array}{c}\text { After } \\
\text { surgery (\%) }\end{array}$ \\
\hline Object naming & 95 & 75 \\
\hline Auditory kana comprehension & 100 & 70 \\
\hline Auditory kanji comprehension & 100 & 100 \\
\hline Word repetition & 100 & 100 \\
\hline Picture explanation & 90 & 90 \\
\hline Kana/picture correspondence & 100 & 0 \\
\hline Kanji/picture correspondence & 100 & 100 \\
\hline Kana reading & 100 & 40 \\
\hline Kanji reading & 100 & 40 \\
\hline Kana writing & 63 & 53 \\
\hline Kanji writing & 77 & 53 \\
\hline
\end{tabular}

Percentages of correct answers are shown

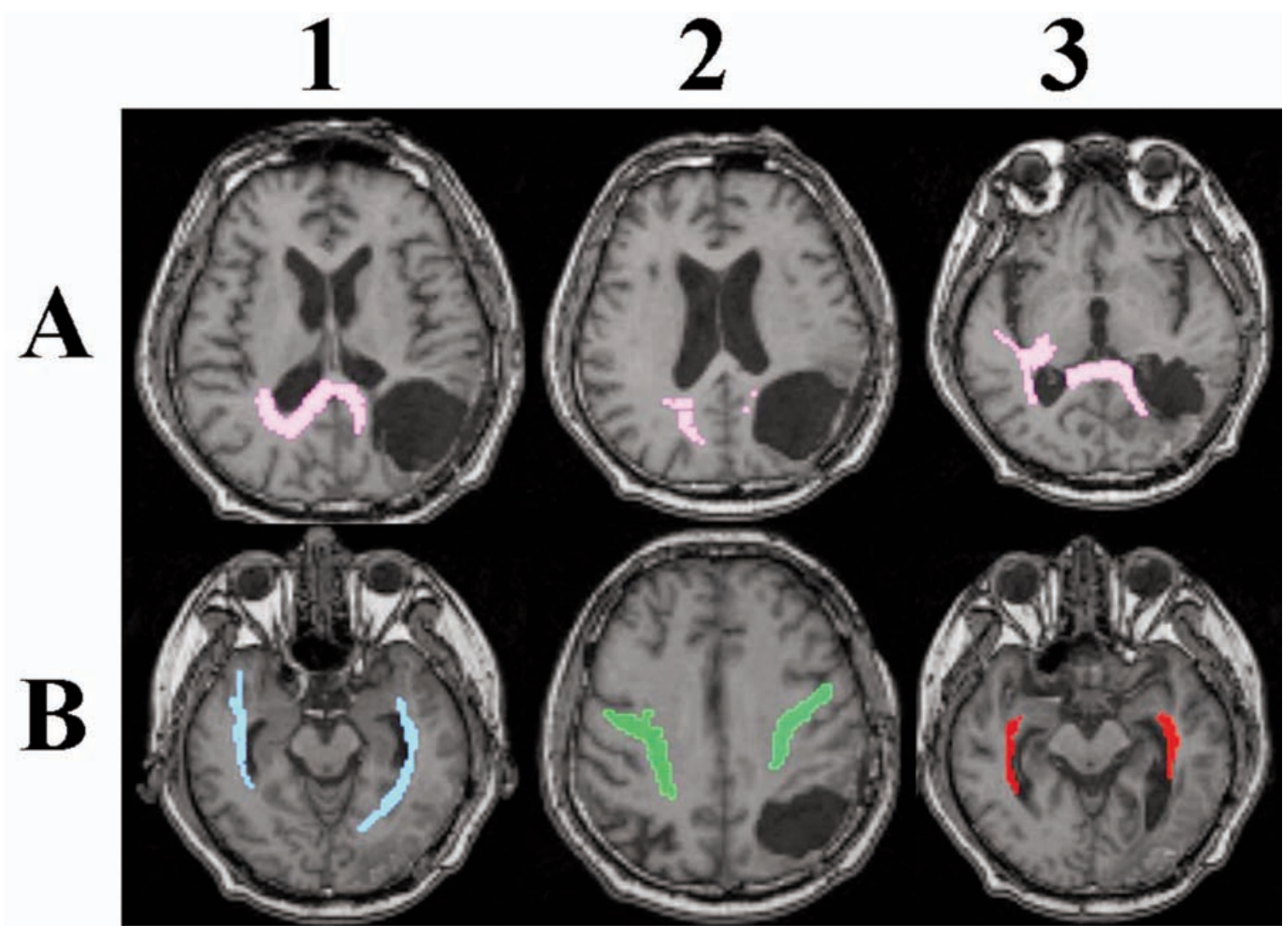

Figure 3: DTI images: Axial views after second surgery. The dorsal OCF (Panel A-1, A-2; pink), ventral OCF (Panel A-3; pink), ILF (Panel B-1; light blue), SLF (Panel B-2; green) and IFOF (Panel B-3; red) were constructed as described in the Methods section. The left dorsal OCF was damaged after surgery, whereas the left ventral OCF, ILF, SLF and IFOF remained intact after surgery. 
comprehension, word repetition, picture explanation, kana or kanji/picture correspondence, kana or kanji reading, and kana or kanji writing were assessed using the SLTA. The Visual Perception Test for Agnosia (VPTA, Tokyo, Japan) was performed to assess perception of objects, pictures, face, color, symbols, visual field and geography.

\section{RESULTS}

\section{DTI studies}

In comparison with MRI imaging performed before surgery (Figure 1A), MRI imaging after surgery demonstrated removal of the deep white matter of the left IPL beside the posterior horn of the lateral ventricle (Figure 1B). Tractography was performed before and after surgery with a focus on the left temporal, parietal and occipital lobe, namely the dorsal and ventral OCF, ILF, SLF, and IFOF, to evaluate the neural tracts damaged by bleeding and/or surgery (Figure 2, 3). The ventral OCF (pink fibers), ILF (light blue fibers), SLF (green fibers), and IFOF (red fibers) were observed before and after surgery in the left hemisphere (Figure 2A-3, 2B-1, 2B-2, 2B-3, 3A-3, 3B-1, 3B-2, $3 \mathrm{~B}-3$ ). By contrast, the dorsal OCF (pink fibers) was observed on the left side before surgery but not after surgery (Figure 2B-1, 2B-2, 2C-1, 2C-2). The results suggest that dorsal OCF damage was present postoperatively.

\section{Neuropsychological findings}

Standard Language Test of Aphasia conducted before and after second surgery demonstrated that kana/picture correspondence deteriorated from 100 to 0 (Table). Similarly, kana and kanji reading deteriorated from 100 to 40. By contrast, object naming, auditory kana or kanji comprehension, word repetition, picture explanation, and kana or kanji writing abilities remained stable over this time. This implies that damage to this area after surgery was associated with a specific deficit in kana/picture correspondence and reading. These indicate severe anomic alexia of kana and moderate alexia of kanji after surgery. VPTA conducted after the second surgery demonstrated that there was deterioration in perception for line tilt, cube drawing, and picture classification. These deficits suggest deterioration of higher-order visual processing.

\section{Discussion}

The present study described a case of severe anomic alexia of kana and moderate alexia of kanji in a patient with damage to the left deep white matter of the IPL, including the dorsal OCF, due to tumor bleeding and surgery. Since the present patient demonstrated an intact left ILF, SLF, IFOF and ventral OCF before and after surgery, these data suggest that compromise of the left white matter of the IPL, including the dorsal OCF, resulted in severe anomic alexia of kana. Of note, the arcuate fasciculus, namely the ventral fibers of the SLF, was not identified before or after surgery, suggesting that this fiber was unlikely to be related to the severe anomic alexia of kana (data not shown).

Investigators have previously utilized fMRI, positron emission tomography (PET) study and lesion analysis in patients to demonstrate that the left posterior inferior or middle temporal cortex is critical for reading or writing of kanji $\mathrm{j}^{2-4,6-8}$. Other investigators have reported that selective kana agraphia can develop after cerebral infarctions in a part of the left angular gyrus, its adjoining superior posterior temporal gyrus, and the corona radiata ${ }^{1}$. Further, studies using PET have shown that the left angular gyrus was activated in a mental kana writing task ${ }^{2}$. In fMRI, the Stroop task activated an area in the left IPL during the kana task ${ }^{4}$. When taken in combination, these observations suggest that that the dorsal route including the IPL is involved in processing of kana tasks, while the central route including the inferior temporal lobe is involved in the processing of kanji tasks $^{3,5}$

In the present case, tumor was present in the IPL before surgery, and the patient did not show any deficit in kana/picture correspondence and kana reading. However, severe impairment of kana/picture correspondence and kana reading (anomic alexia of kana) was present after surgery, which corresponded with MRI-documented removal of the deep white matter in the IPL (Figure 1). It is to be noted that as the patient was not tested between hemorrhage and the second surgery, it is not possible to determine definitively whether the deficit was due to hemorrhage into the parietal white matter and cortex or the surgery. However, since we removed only hemorrhage which contained the tumor by surgery, damage of deep white matter in the IPL was possibly induced by hemorrhage. Regardless, these results indicate that anomic alexia of kana is associated with the deep white matter of the IPL rather than the cortex alone. Imaging studies showed stable results when comparing testing performed two to three weeks postoperatively and two months postoperatively, suggesting that this phenomenon was not due to postoperative edema or inflammation. Of the neural tracts characterized in this study, the dorsal OCF was the only one that sustained damage. Interestingly, anomic alexia of kanji can develop after damage to the middle part of the left middle temporal gyrus and its subcortical area, suggesting that some common mechanism may underlie both kanji naming and kanji reading ${ }^{9}$. Similarly, anomic alexia of kana may be related to damage to the IPL and its subcortical area.

Damage to the splenium results in impairments in picture naming and oral reading or alexia without agraphia. This is because the right hemisphere plays a role in reading and because disconnection of callosal pathways results in impairments in reading ${ }^{10,18,19}$. Pure alexia for kana is associated with lesions of the occipital cortex, and pure alexia for Danish-language words is related to abnormal function of visual processing, suggesting that lesions in the occipital lobe play a role in pure alexia for both words ${ }^{20-22}$. Indeed, this patient suffered from higher-level visual processing deficit. As demonstrated by tractography and MRI, the ventral and dorsal OCF run through the splenium from the occipital lobe ${ }^{23,24}$. The dorsal visual maps (dorsal to calcarine) send projections through a large band in the middle of the splenium, while the ventral visual maps send projections through the inferior-anterior corner of the splenium. Callosal pathways mediating reading run above the occipital horn, which suggests that the dorsal OCT may play a critical role in reading ${ }^{25}$. Kana and English-language words may be mediated through similar dorsal routes because both are phonographic words. Therefore, these data are consistent with the hypothesis that damage to the dorsal OCT may mediate alexia of phonographic words, namely kana. 


\section{ACKNOWLEDGMENTS}

The authors thank H. Shinoura for assistance with manuscript preparation.

\section{REFERENCES}

1. Tanaka Y, Yamadori A, Murata S. Selective Kana agraphia: a case report. Cortex. 1987;23:679-84.

2. Tokunaga H, Nishikawa T, Ikejiri Y, Nakagawa Y, Yasuno F, Hashikawa K, et al. Different neural substrates for Kanji and Kana writing: a PET study. Neuroreport. 1999;10:3315-9.

3. Thuy DH, Matsuo K, Nakamura K, Toma K, Oga T, Nakai T, et al. Implicit and explicit processing of kanji and kana words and non-words studied with fMRI. Neuroimage. 2004;23:878-89.

4. Coderre EL, Fillippi CG, Newhouse PA, Dumas JA. The Stroop effect in kana and kanji scripts in native Japanese speakers: a fMRI study. Brain Lang. 2008;107:124-32.

5. Koyama MS, Hansen PC, Stein JF. Logographic Kanji versus phonographic Kana in literacy acquisition: how important are visual and phonological skills. Ann N Y Acad Sci. 2008;1145: 41-55.

6. Kawamura M, Hirayama K, Hasegawa K, Takahashi N, Yamaura A. Alexia with agraphia of kanji (Japanese morphograms). J Neurol Neurosurg Psychiatry. 1987;50:1125-9.

7. Soma Y, Sugishita M, Kitamura K, Maruyama S, Imanaga H. Lexical agraphia in the Japanese language. Pure agraphia for Kanji due to left posteroinferior temporal lesions. Brain. 1989;112:1549-61.

8. Sakurai Y, Mimura I, Mannen T. Agraphia for kanji resulting from a left posterior middle temporal gyrus lesion. Behav Neurol, 2008; 19:93-106.

9. Yamawaki R, Suzuki K, Tanji K, Fujii T, Endo K, Meguro K, et al. Anomic alexia of kanji in a patient with anomic aphasia. Cortex. 2005;41:555-9.

10. Marsh EB, Hillis AE. Cognitive and neural mechanisms underlying reading and naming: evidence from letter-by-letter reading and optic aphasia. Neurocase. 2005;11:325-37.

11. Catani M, Jones DK, Donato R, Ffytche DH. Occipito-temporal connections in the human brain. Brain. 2003;126:2093-107.

12. Rauschecker AM, Deutsch GK, Ben-Shachar M, Schwartzman A, Perry LM, Dougherty RF. Reading impairment in a patient with missing arcuate fasciculus. Neuropsychologia. 2009;47:180-94.
13. Shinoura N, Suzuki Y, Yamada R, Kodama T, Takahashi M, Yagi K. Fibers connecting the primary motor and sensory area play a role in grasp stability of the hand. Neuroimage. 2005;25:936-41.

14. Reese TG, Heid O, Weisskoff RM, Wedeen VJ. Reduction of eddycurrent-induced distortion in diffusion MRI using a twicerefocused spin echo. Magn Reson Med. 2003;49:177-82.

15. Basser PJ, Mattiello J, LeBihan D. MR diffusion tensor spectroscopy and imaging. Biophys J. 1994;66:259-67.

16. Conturo TE, Lori NF, Cull TS, Akbudak E, Snyder AZ, Shimoi JS, et al. Tracking neuronal fiber pathways in the living human brain. Proc Natl Acad Sci USA. 1999;96:10422-7.

17. Mimura M, Kato M, Kato M, Sano Y, Kojima T, Naeser M, et al. Prospective and retrospective studies of recovery in aphasia. Changes in cerebral blood flow and language functions. Brain. 1998; 121:2083-94.

18. Larsen J, Baynes $\mathrm{K}$, Swick D. Right hemisphere reading mechanisms in a global alexia patient. Neuropsychologia. 2004; 42:1459-76.

19. Mao-Draayer Y, Panitch H. Alexia without agraphia in multiple sclerosis: case report with magnetic resonance imaging localization. Mult Scler. 2004;10:705-7.

20. Sakurai Y, Terao Y, Ichikawa Y, Ohtsu H, Momose T, Tsuji S, et al. Pure alexia for kana. Characterization of alexia with lesions of the inferior occipital cortex. J Neurol Sci. 2008;268: 48-59.

21. Sheldon CA, Malcolm GL, Barton JJ. Alexia with and without agraphia: an assessment of two classical syndromes. Can J Neurol Sci. 2008;35:616-24.

22. Starrfelt R, Habekost T, Gerlach C. Visual processing in pure alexia: a case study. Cortex. 2009 [Epub ahead of print].

23. Dougherty RF, Ben-Shachar M, Brammer R, Brewer AA, Wandell BA. Functional organization of human occipital-callosal fiber tracts. Proc Natl Acad Sci USA. 2005;102:7350-5.

24. Dougherty RF, Ben-Shachar M, Deutsch G, Potanina P, Bammer R, Wandell BA. Occipital-callosal pathways in children: validation and atlas development. Ann N Y Acad Sci. 2005;1064:98-112.

25. Binder JR, Mohr JP. The topography of callosal reading pathways. A case-control analysis. Brain. 1992;115:1807-26. 